\title{
Probiotics for infectious diarrhoea
}

\section{E Isolauri}

$\mathrm{T}$ he modern view of probiotic therapy derives from the concept of a well functioning gut barrier and a normal balanced microbiota. The scientific conception of these host defences has changed; the gut barrier was seen primarily as a physical blockade to foreign antigen entry until its active role in shaping the immune response to luminal antigens was deciphered. Concomitantly, recent experimental and clinical studies demonstrate a dependency on healthy host-microbe interaction to cope with antigen challenges, and consequently attribute the gut microbiota an active role in barrier function..$^{1-3}$

The most fully documented probiotic intervention is the treatment of acute infectious diarrhoea. Well controlled clinical studies have shown that probiotics such as Lactobacillus rhamnosus $G G, L$ reuteri, $L$ casei Shirota, and Bifidobacterium lactis $\mathrm{Bb} 12$ can shorten the duration of acute rotavirus diarrhoea. ${ }^{45}$ Numerous publications on different populations have since substantiated the success of specific probiotic strains in the adjunct management of viral diarrhoea, the main goal still being restoration of fluid and electrolyte losses. To combine these approaches, a multicentre study by the European Society for Paediatric Gastroenterology, Hepatology, and Nutrition working group tested the clinical efficacy and safety of a probiotic administered in an oral rehydration solution. ${ }^{6}$ In rotavirus diarrhoea, a significant curtailment of episodes was observed, while in non-specific or bacterial diarrhoea no clear effect was found.

The potential mechanisms by which probiotics fight infectious diarrhoea include exclusion of pathogens by means of competition for binding sites and available substrates, lowering of luminal $\mathrm{pH}$ and production of bacteriocins, and promotion of the production of mucus - that is, commensal pathogen cross talk (fig 1). The capacity of pathogenic bacteria to produce mucinase may indeed partly explain the superiority of probiotic therapy in viral diarrhoea. ${ }^{6}$ Enhancement of intestinal motility and upregulation of genes mediating innate immunity by probiotics $^{7}$ further contribute to the eradication of pathogens. Lactobacilli and bifidobacteria produce short chain fatty acids which regulate cell growth and differentiation and have trophic effects on the intestinal epithelium, and additionally stimulate bifidobacteria and counteract urease producing strains, thus maintaining gut barrier function. A lactobacillus strain isolated from human microbiota was recently shown to counter cellular damage associated with a diarrhoeagenic pathogen, thus corroborating reports of a normalising of intestinal permeability by selected probiotics. ${ }^{8}$ Furthermore, specific probiotic strains balance the generation of pro- and anti-inflammatory cytokines, thereby creating the healthy host-microbe cross talk (fig 1) needed to keep inflammatory responses in check but concomitantly readily primed. Sustained inflammatory activation is required for immunological surveillance at the major portal of entry of pathogens, while the antiinflammatory effect of specific strains of gut microbiota is

Department of Paediatrics, University of Turku, 20520 Turku, Finland; erika.isolauri@utu.fi

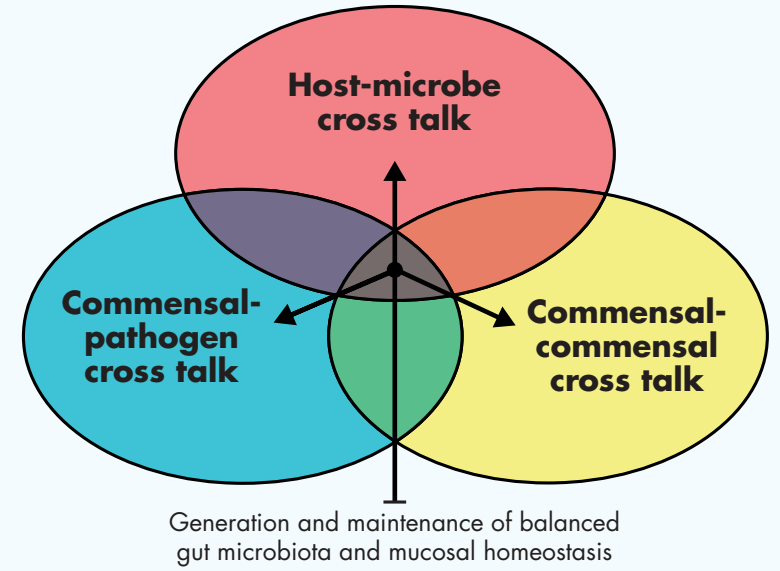

Figure 1 Selections of specific strains for the generation and maintenance of balanced gut microbiota and mucosal homeostasis.

requisite to prevent the evolution of infectious diarrhoea toward a protracted course or other chronic sequelae such as allergic and autoimmune disease. ${ }^{3}$

While it appears evident that balanced normal microbiota may become aberrant and immunogenic secondary to gut related disease such as infectious diarrhoea, and thus constitute a target for probiotic therapy, it is not known whether aberrances in the composition of the microbiota can be a primary cause of disease. Predisposition to infectious diarrhoea is associated with immaturity and dysfunction of the gut barrier, and establishment of the gut microbiota provides the critical maturational signals. ${ }^{1-3}$ Here the

\section{Summary}

(1) Probiotics have been variously defined according to their initial application in animal feeds. For the purpose of human nutrition, a probiotic is defined as a live microbial food ingredient beneficial to health.

(2) Specific probiotic strains have been shown to normalise increased intestinal permeability and altered gut microecology, to promote intestinal barrier functions, and to alleviate the intestinal inflammatory response.

(3) To date, the most fully studied clinical application of probiotics, principally lactobacilli and bifidobacteria, is as dietary supplements for the treatment of acute infantile diarrhoea.

(4) The normal gut microbiota still represents an unexplored ecosystem, with micro- organisms carrying distinct pro- and anti-inflammatory effects.

(5) Current probiotic research aims at characterisation of the gut microbiota as a model for selected natural or engineered probiotic strains and a fuller understanding of microbe-microbe and host-microbe interactions within the gastrointestinal tract. 


\section{THERAPY UPDATE}

collective composition of the colonising strains may be crucial. Specific probiotics have proved effective in preventing acute infantile diarrhoea, ${ }^{4}$ and specific strains of the gut microbiota even of the same genus have different immunomodulatory effects. ${ }^{9}$ There may thus be a third angle to host-microbe cross talk: overall balance within the gut microbiota (fig 1). Identification of the immunomodulatory mechanisms of discrete strains might be exploited in the future development of probiotics for specific prophylactic and therapeutic interventions not only for different infectious diarrhoeas but also for chronic inflammatory diseases.

\section{REFERENCES}

1 Cebra JJ. Influences of microbiota on intestinal immune system development. Am J Clin Nutr 1999;69(suppl): 1046-51.

2 Grönlund $M M$, Arvilommi $H$, Kero $P$, et al. Importance of intestinal colonisation in the maturation of humoral immunity in early infancy: a prospective follow up study of healthy infants aged 0-6 months. Arch Dis Child 2000;83:F186-92.
3 Isolauri E, Rautava S, Kalliomäki $M$, et al. Role of probiotics in food hypersensitivity. Curr Opin Immunol Clin Allergol 2002;2:263-71. 4 Szajewska H, Mrukowicz JZ. Probiotics in the treatment and prevention of acute infectious diarrhea in infants and children: a systematic review of published randomized, double-blind, placebo-controlled trials. J Pediatr Gastroenterol Nutr 2001;33:S17-25.

5 Isolauri E, Kirjavainen PV, Salminen S. Probiotics-a role in the treatment of intestinal infection and inflammation? Gut 2002; 50(suppl III):54-9

6 Guandalini S, Pensabene L, Zikri MA, et al. Lactobacillus GG administered in oral rehydration solution to children with acute diarrhoea: a multicenter European trial. J Pediatr Gastroenterol Nutr 2000:30:54-60

7 Neish AS, Gewirtz AT, Zeng $\mathrm{H}$, et al. Prokaryotic regulation of epithelial responses by inhibition of IkappaB-alpha ubiquitination. Science 2000;289: 1560-63.

8 Liévin-Le Moal V, Amsellem R, Servin AL, et al. Lactobacillus acidophilus (strain LB) from the resident adult human gastrointestinal microflora exerts activity against brush border damage promoted by a diarrhoeagenic Escherichia coli in human enterocyte-like cells. Gut a diarrhoeagenic

9 He F, Morita H, Hashimoto H, et al. Intestinal bifidobacterium species induce varying cytokine production. J Allergy Clin Immunol 2002;109:1035-6. 